
LA-UR-
$91-3239$

LA-UR- $-91-3239$

$1 / 8--25$

DE92 002440

Los Alamos National Laboratory is operated by the University of Califomia for the United States Department of Energy under contract W-7405-ENG-36

\title{
TITLE: WIGGLER FIELD MEASUREMENTS AND CORRECTIONS USING THE PULSED WIRE TECHNIQUE
}

AUTHOR(S): Daryl W. Preston and Roger W. Warren

\author{
SUBMITTED TO: 13th International Free Electron Laser Conference \\ Santa Fe, NM \\ August 25-30, 1991
}

\section{DISCLAIMER}

\begin{abstract}
This report was prepared as an account of work sponsored by an agency of the United States Government. Neither the United States Government nor any agency thereof, nor any of their employees, makes any warranty, express or implied, or assumes any legal liability or responsibility for the accuracy, completeness, or usefulness of any information, apparatus, product, or process disclosed, or represents that its use would not infringe privately owned rights. Reference herein to any specific commercial product, process, or service by trade name, trademark, manufacturer, or otherwise does not necessarily constitute or imply its endorsement, recommendation, or favoring by the United States Government or any agency thereof. The views and opinions of authors expressed herein do not necessarily state or reflect those of the United States Government or any agency thereof.
\end{abstract}




\title{
WIGGLER FIELD MEASUREMENTS AND CORRECTIONS \\ USING THE PULSED WIRE TECHNIQUE
}

\author{
Daryl W. Preston* \\ Roger W. Warren \\ Los Alamos National Laboratory \\ Los Alamos, NM 87545
}

We have performed magnetic field measurements on permanentmagnet wigglers using the pulsed wire technique. The first and second integrals of the field were directly measured, and the field was determined by differentiating the first integral. The pulsed wire technique permits the rapid accumulation of data and the identification of defective wiggler magnets. Magnet defects of different types can be distinguished and compensated for by attaching small, appropriately oriented magnets to each defective magnet. We have made major improvements in the fields using these techniques.

*Permanent address: Physics Department, California State University, Hayward, CA 94542. Work supported by the Los Alamos National Laboratory Institutional Supporting Research, under the auspices of the United States Department of Energy and the Division of Advanced Energy Projects, Ciffice of Basic Energy Sciences. 


\section{NTRODUCTION}

The fabrication of a permanent-magnet wiggler is a complicated process that involves selecting individual magnets, assembling the magnets, testing the fully assembled wiggler, and finally, correcting the assembled wiggler for imperfect individual magnets. Individual magnets are usually measured for strength, before being selected for assembly. Magnets that deviate too far from an average strength, must be discarded. Assembly of the wiggler involves proper placement of selected magnets to ensure uniform amplitude of the wiggler field; that is, the variations in field strength of individual magnets should compensate one another. Testing and correcting the assembled wiggler are the topics of this paper.

Ideally, the magnetic moment of each small part of a magnet should be constant in magnitude, parallel to all other parts, and oriented perpendicular to the long axis of the magnet. Deviations from this ideal case give rise to magnet, we find imperfections, which we have encountered. To identify imperfect magnets, we find it is especially convenient to measure the second integral of the fields, both on and off the wiggler axis, that are orthogonal to the wiggler axis. Using a Hall probe for such measurements is very time consuming because it is necessary to measure the vertical field along the wiggler axis, integrate it twice, then rotate the probe $90^{\circ}$, measure the horizontal field along the axis, integrate twice, and finally, repeat these measurements off-axis. A single measurement using the pulsed wire technique, which requires a few milliseconds, is sufficient to establish the second integral of both the horizontal and vertical fields. The wire may then be displaced off-axis and the second integral measured as a 
function of wire displacement. Once the defective magnets are identified and characterized, they can be quickly corrected and remeasured.

In the sections below, the following topics are discussed:

(1) the apparatus used in the pulsed wire technique, (2) measurement of the field and measurements of the first and second integrals of the field, (3) locating bad magnets, (4) analyses of field errors, and (5) correction of field errors.

\section{APPARATUS}

What follows is a discussion of the apparatus we used in the pulsed wire technique. For additional information, see Reference 1. As shown in figure 1, a 2-mil tungsten wire is threaded through the wiggler along the $z$ axis. Laser diodes [2], which generate $4.25 \mathrm{~mW}$ at $670 \mathrm{~nm}$, are convenient, compact light sources, and photodiodes [3] are fast photodetectors. Orthogonal optical detectors, as shown in figure 1, are necessary to identify error fields in both the $\mathrm{x}$ and $\mathrm{y}$ directions. Each laser-photodiode pair is mounted on a translation stage and moved relative to the wire's equilibrium position (wire displacement $d=0$ ) until the sensor's output voltage is one-half of its maximum value. The output voltage at this point is approximately a linear function of $d$. The wire forms the collector resistance of a power transistor. A $60-\mathrm{V}$ pulse of variable width excites a wave on the wire proportional to the wiggler's field strength.

\section{FIELD MEASUREMENTS}

Figures $2 a-2 c$ show the wiggler field, the first integral of the field, and the second integral of the field, respectively, for a $2.7-\mathrm{cm}$ tapered- 
period wiggler. The wire used for the measurement was centered in the wiggler. If the current pulse in the wire is short (about $20 \mu \mathrm{s}$ ), the resulting signal (figure $2 b$ ) is the first integral of the field [1]. The peak-to-peak amplitude in figure $2 \mathrm{~b}$ increases to the right because of tapering; that is, the wiggler period increases to the right. The field shown in figure $2 a$ is determined by differentiating numerically the first integral, shown in figure $2 b$. Neither the field nor the first integral is very sensitive to imperfections in the wiggler magnets. Spurious signals may be present due to wire imperfections. Such signals are discussed in Reference 4.

The second integral of the field, as shown in figure $2 \mathrm{c}$, is recorded by using a long (few milliseconds) current pulse [1]. The second integral is particularly sensitive to individual magnet errors. The second integral is also particularly useful because it shows the trajectory followed by the electron beam [1]. The $1-\mathrm{mm}$ scale on figure $2 \mathrm{c}$ indicates the radius of the electron beam in a typical experiment. To maximize gain, the beam trajectory should wander no more than about $10 \%$ of the beam radius. As can be seen in figure $2 c$, however, the variation in beam trajectory in the wiggler is excessive and must be reduced. The other signal shown in figure $2 c$ is caused by magnet field errors in the $x$ direction. This signal variation is also excessive. We will address this matter in the next section.

\section{LOCATING BAD MAGNETS}

The field in the $y$, or vertical, direction produces the oscillatory second integral signal (called the major signal), and the field in the $x$, or horizontal, direction produces the deflections of the other trace shown 
in figure $2 \mathrm{c}$ (called the minor signal). A change in the slope of the centroid of either trace identifies the location of an imperfect wiggler magnet. Consider the major signal in figure $2 \mathrm{c}$, for example. The initial positive slope of the centroid is caused by a bad adjustment of the lefthand steering magnet (adjustable magnets are at both ends of the wiggler). The slope then changes abruptly at the 7 th wiggler period (28th magnet), the 12th period (48th magnet), the 19th period (76th magnet), and the 22nd period (88th magnet), and then gradually changes from the 31 st through the 35 th periods. Each point where the slope changes identifies an imperfect magnet.

The minor signal also shows slope changes at defective magnets. This signal has a large change in slope at the 19th period, and a gradual change in slope from the $31 \mathrm{st}$ through the 35 th periods. There are also minor changes in the slope before the 19th period. Clearly, there is a correlation between the error fields in the $\mathrm{x}$ and $\mathrm{y}$ directions.

\section{ANALYSIS OF FIELD ERRORS}

Our model for an imperfect magnet contains one or more localized regions with magnetic moments that are misaligned, presenting error moments either parallel or perpendicular to the long axis of the imperfect magnet.

Figure 3 shows a diagram of an imperfect wiggler magnet having a localized magnetic moment (chosen to be centered in the magnet) aligned in the vertical direction. The angular deflection of an electron beam as a function of displacement $d$, when affected by this magnet, is sketched for both the major and minor signals. (Angles $\theta$ and $\phi$ are the deflection angles for the major and minor signals, respectively.) In 
contrast, an imperfect magnet having a localized horizontal moment would produce an antisymmetric $\theta$ versus $d$ curve, and a symmetric $\phi$ versus $d$ curve. These two models of imperfect magnets are used to interpret measured deflection angles and to provide a way to correct magnet errors. The second integral signals are measured as a function of $z$ and for several values of $d$. For each discontinuity along the signals, the changes in slope, $\theta$ and $\phi$, are measured and plotted as a function of d. Such plots are then matched to the models of local vertically or horizontally aligned moments. This process gives the polarization, strength, and position of the magnetization error.

\section{CORRECTION OF FIELD ERRORS}

Our correction procedure involves gluein: to the top of each defective magnet a small correcting magnet with strength equal to that of the magnetization error. The position of this correcting magnet should be as close as possible to the position of the magnetization error, and its polarization should be oppositely oriented. This correcting procedure is only approximate for two reasons: the correcting magnets cannot be positioned in exactly the same place as the magnetization error; and the magnetization errors are not completely localized, as we assume in our model. Therefore, defective magnets need many small correcting magnets for the best results.

As an example of our results, figure $4 \mathrm{a}$ shows the major and minor signals for three values of $d$ before applying correction magnets. Figure $4 \mathrm{~b}$ shows the same signals for the same values of $d$ but with eight vertically polarized correcting magnets attached to magnets number $28,48,56,80,89,121,135$, and 143 and with two horizontally 
polarized magnets attached to the 73rd and 146th magnets. The 76th magnet was particularly difficult to correct because of its extensive and distributed errors. The best correction, as shown in figure $4 \mathrm{~b}$, was obtained by placing one correcting magnet on each side of the defectove 76th magnet. Specifically, a vertically polarized correcting magnet was placed on the 80th magnet, and a horizontally polarized one was placed on the 73rd magnet. Perhaps the best solution would be to replace this especially bad magnet.

The ten magnets specified above improved the electron beam trajectory by a factor of 3 for $d=0$. The result obtained for $d$ values ranging from $-2 \mathrm{~mm}$ to $+2 \mathrm{~mm}$ was improved by a factor of 2 . More than ten magnets give further improvement. Correction is considerably easier for the on-axis $(d=0)$ wiggler field than for the off-axis field; that is, it is easier to correct the field at one point than at multiple points. Correcting the on-axis field improves the trajectory followed by the electron beam. The correction of the field for $d \neq 0$ improves beam focussing by the wiggler.

\section{CONCLUSIONS}

The pulsed wire apparatus is simple and reliable. This experimental sechnique measures values of the field, first intagral of the field, and second integral of the field. Errors in the field may be rapidly identified with particular magnets and corrected by glueing correcting magnets in place. Both on-axis and off-axis fields can be measured and corrected; hence, we can improve both beam trajectory and beam focussing. 


\section{REFERENCES}

[1] R. W. Warren, Nucl. Instr. and Meth. A272 (1988) 257.

[2] LAS-200 Visible Diode Laser. LaserMax, Inc., 207 Tremont Street, Rochester, NY 14608-2303.

[3] OP913SL PIN Si Photodiode. OPTEK Technology, Inc., 1215 Crosby Road, Carrollton, TX 75006.

[4] R. W. Warren and Daryl W. Preston, Magnetic Field Measurements Using the Pulsed Wire Technique, paper delivered at the Thirteenth International Free-Electron Laser Conference, August 26-30, 1991.

\section{FIGURE CAPTIONS}

Figure 1. Simplified diagram of the pulsed wire apparatus.

Figure 2. (a) Wiggler field, (b) first integral of the field, (c) second integral of the field.

Figure 3. Imperfect magnet and the resulting angular deflection of the major and minor signals.

Figure 4. Major and minor signals as a function of displacement $d$ for (a) uncorrected wiggler and (b) corrected wiggler. 


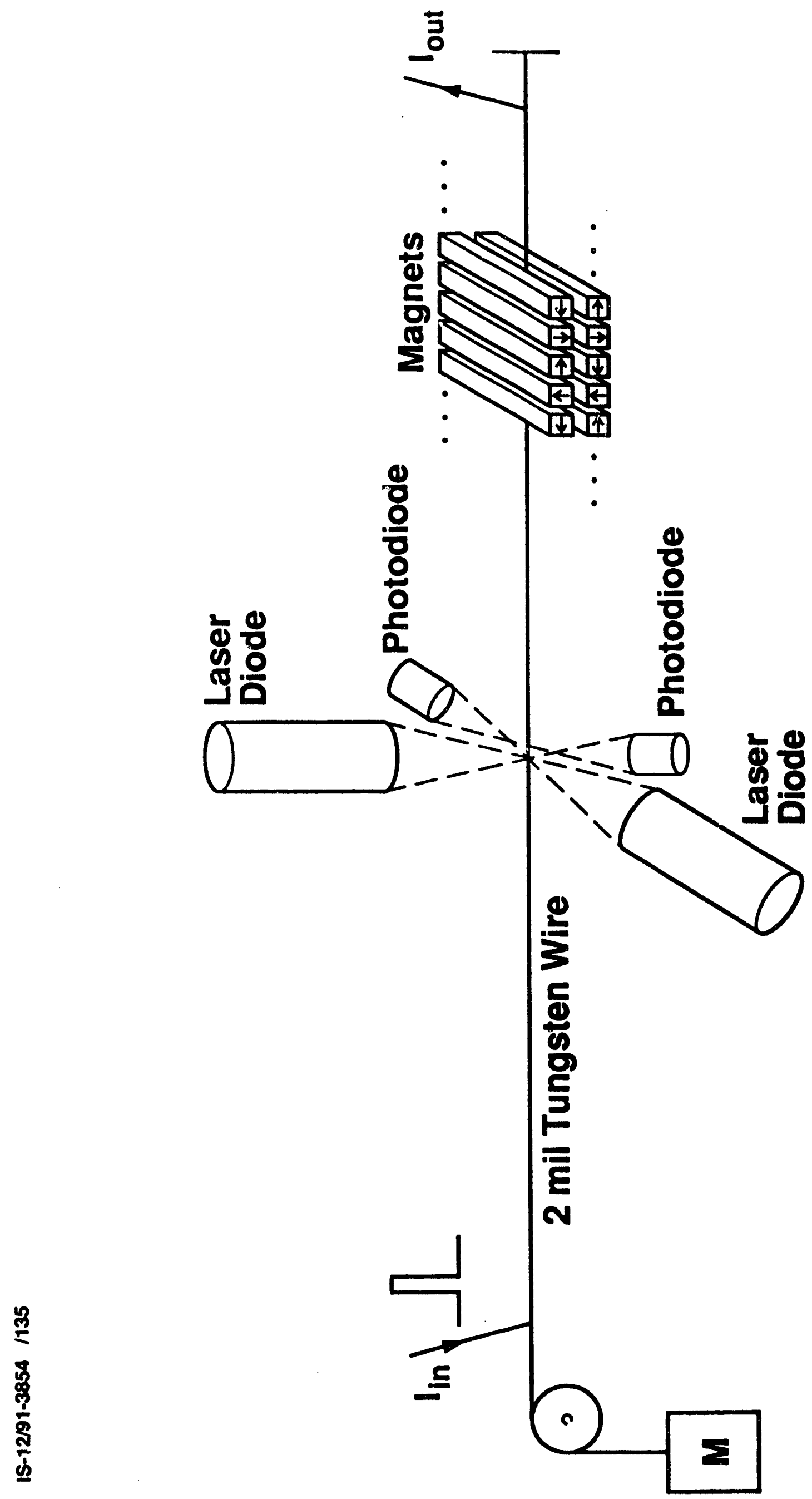


Fig $2 a$

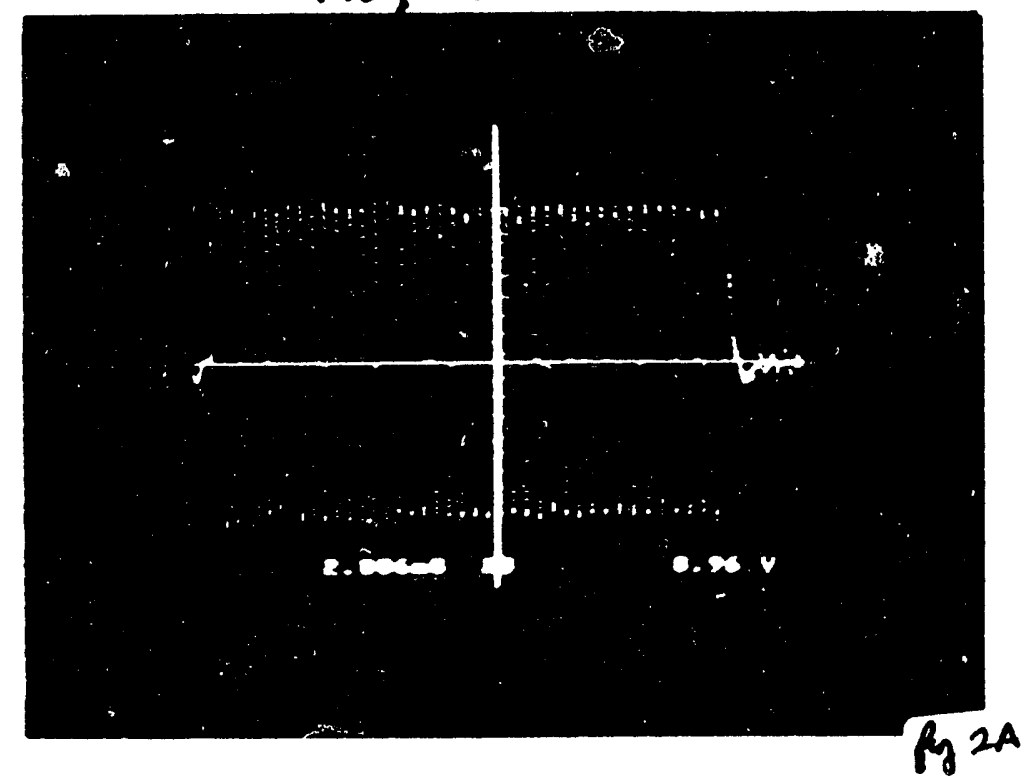




\section{Fis $2 b$}

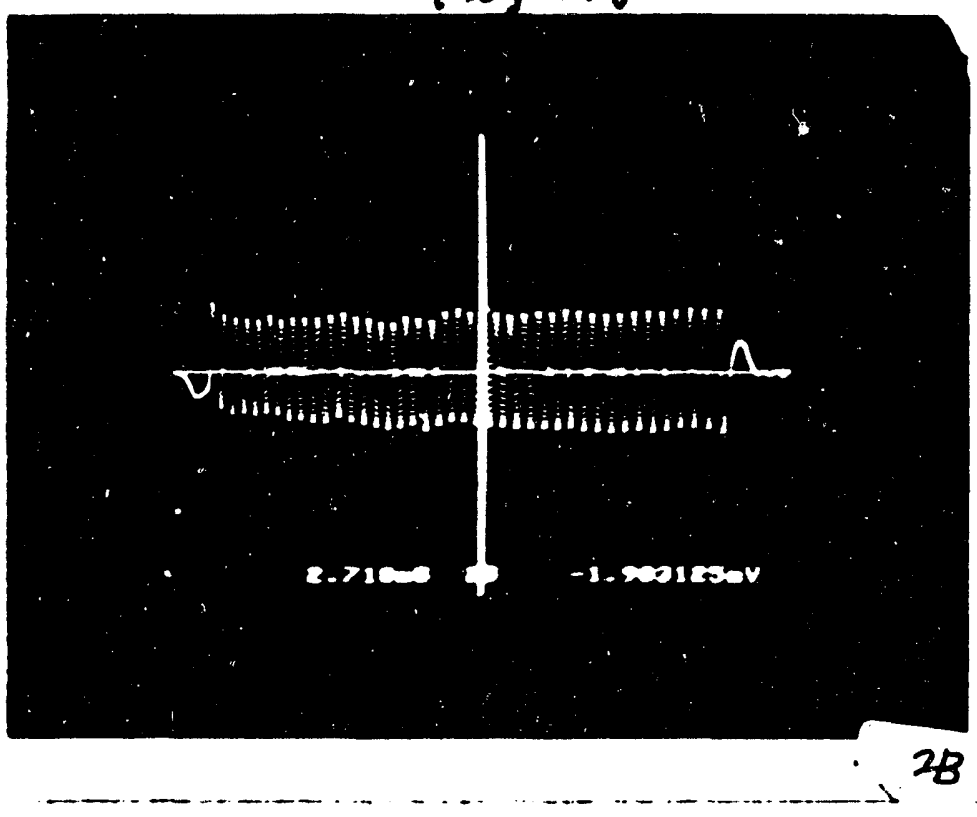




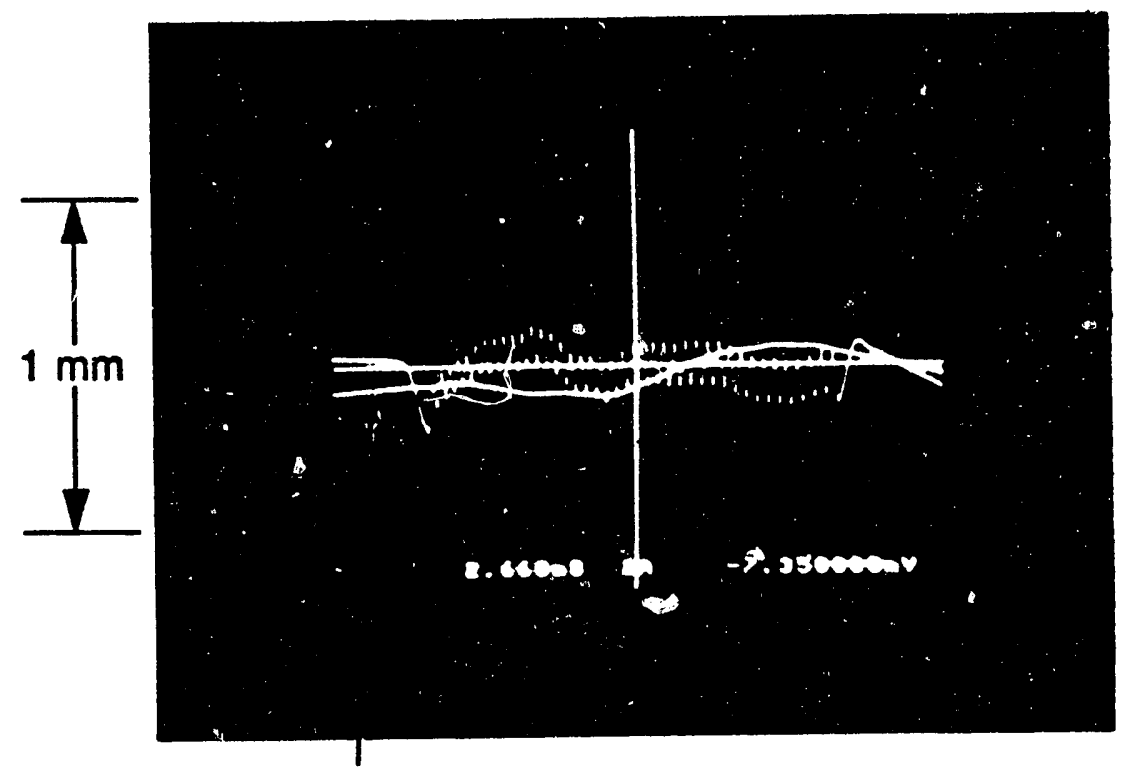

Figure 2c 

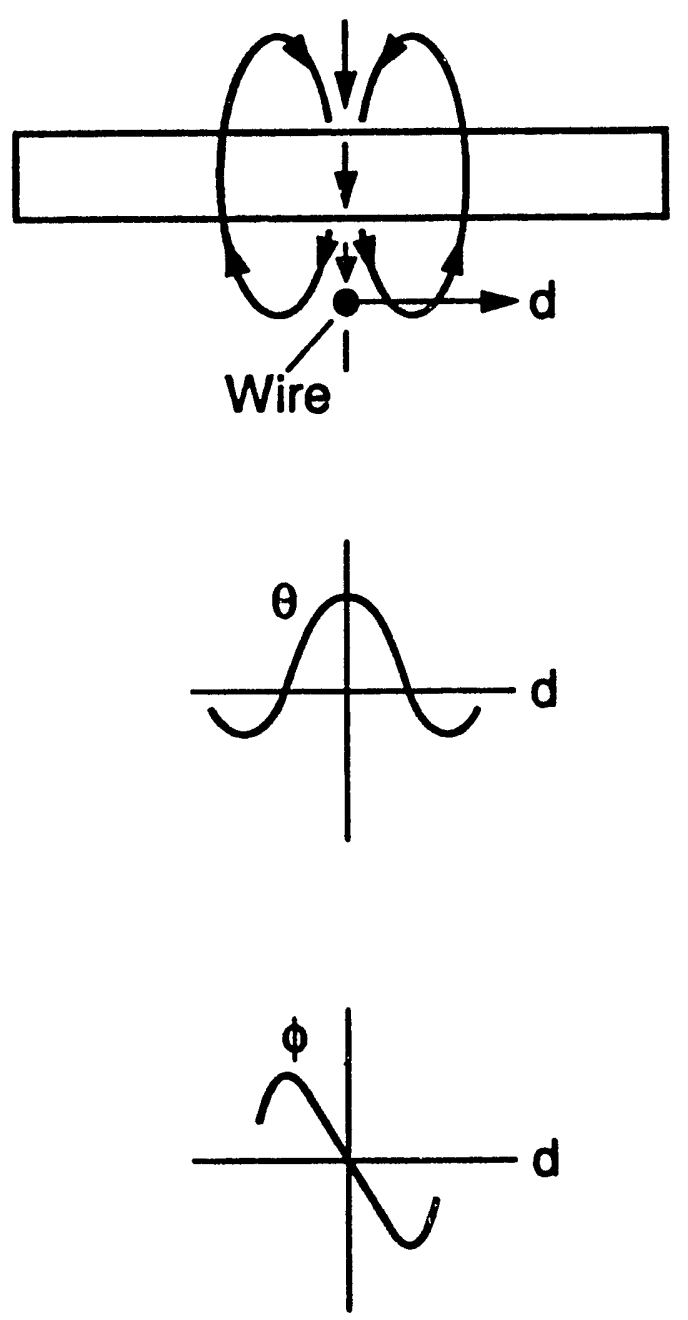

Figure 3 


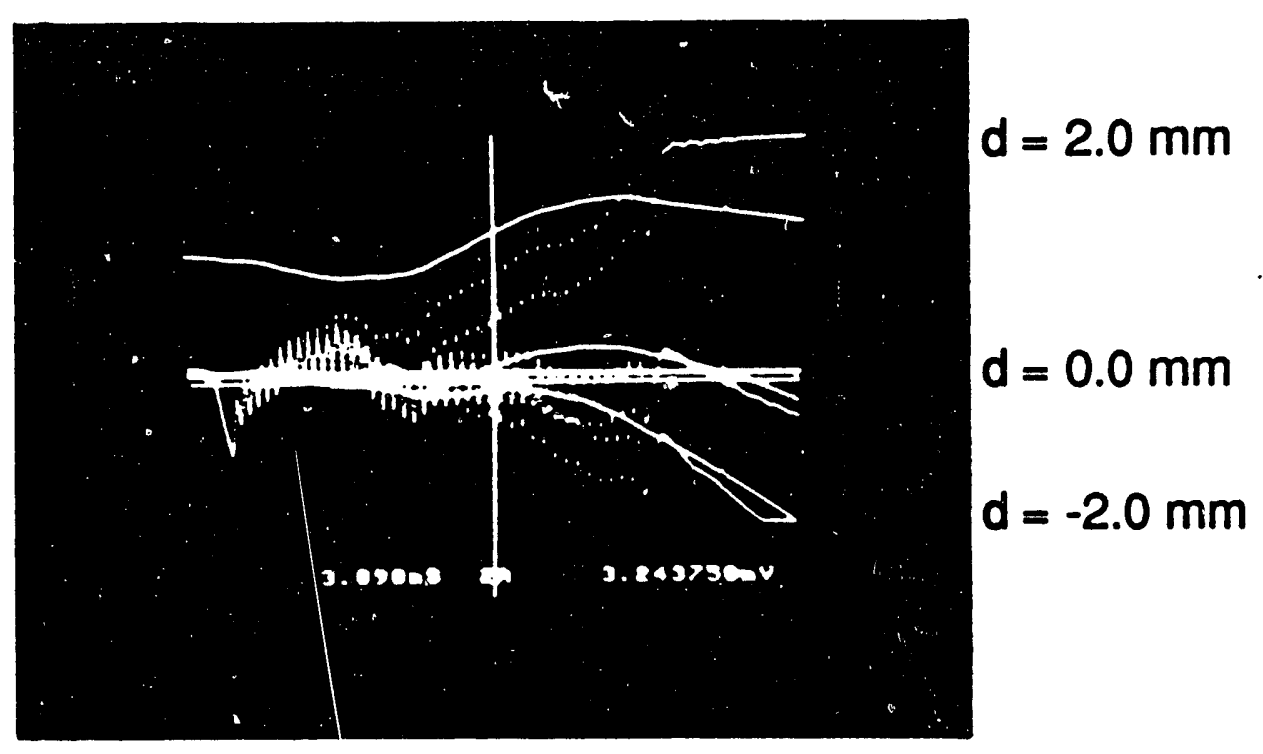

Figure 4a 


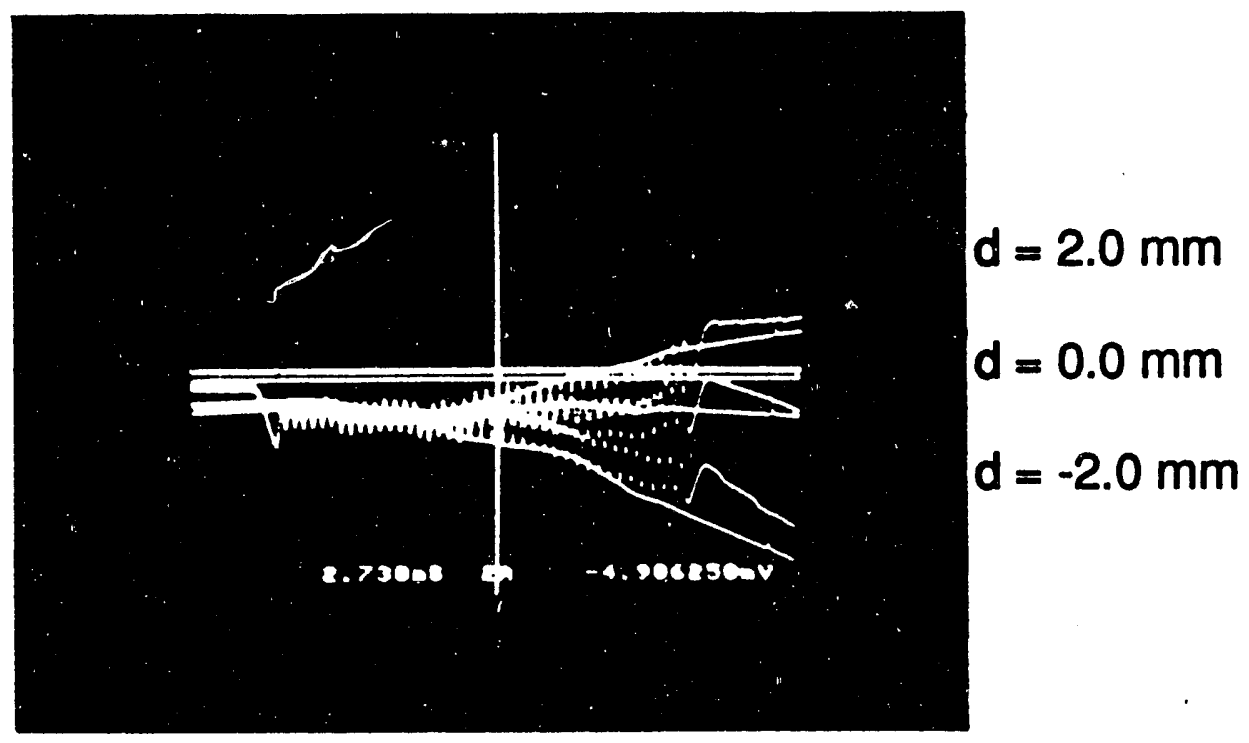

Figure 4b 

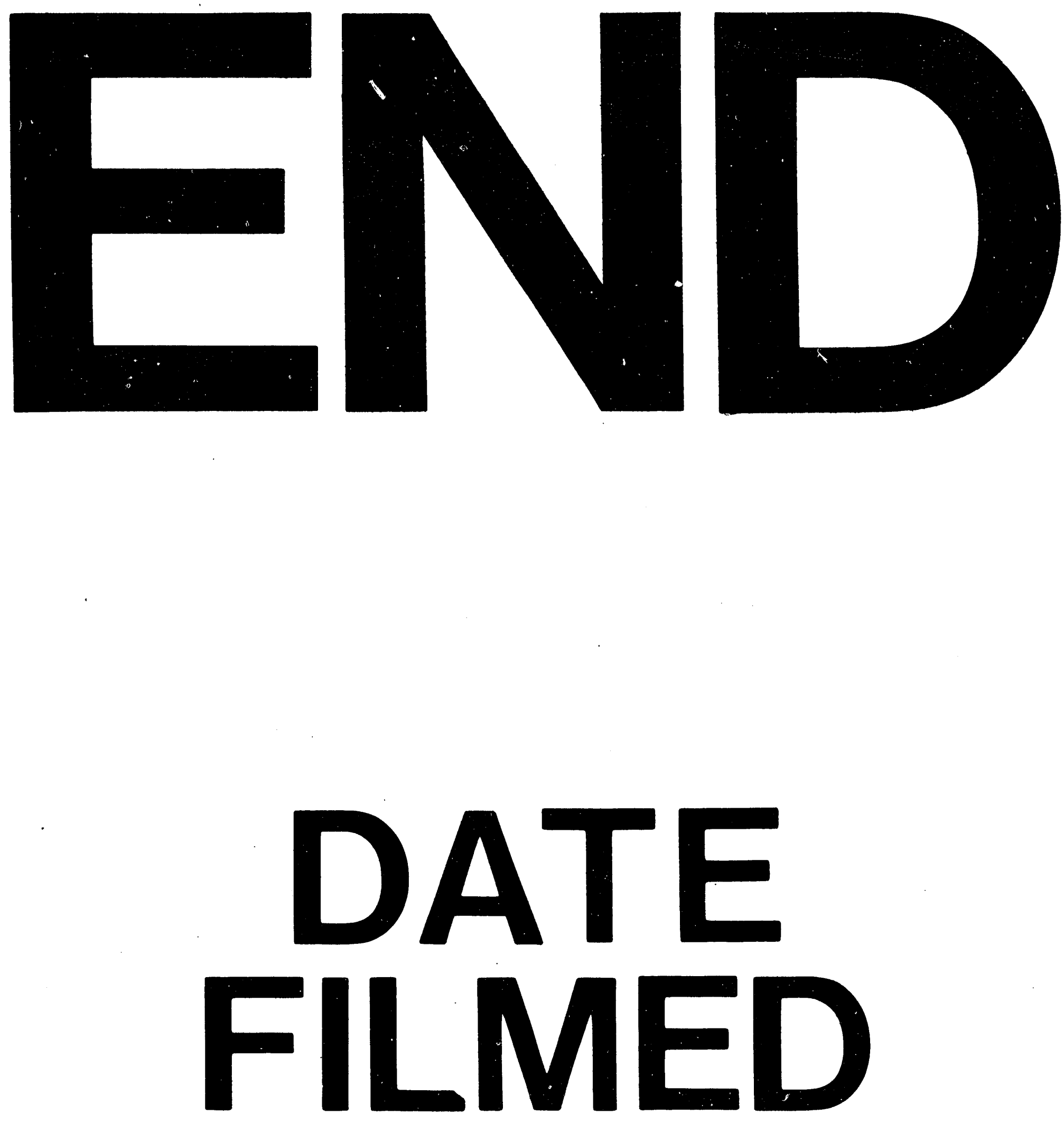

I

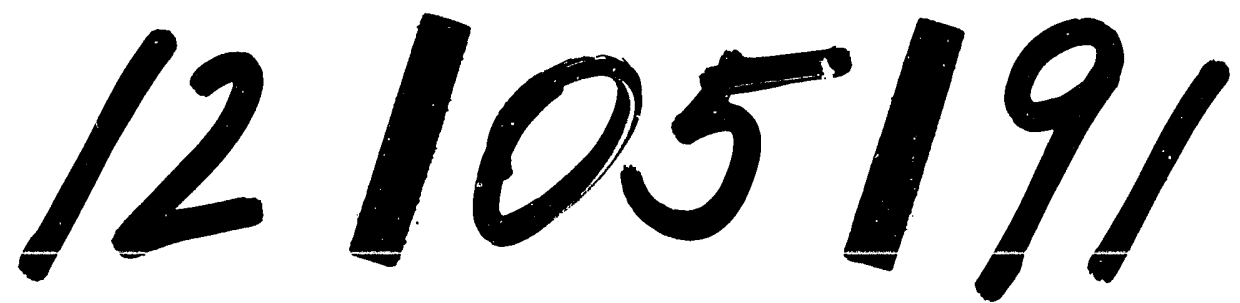


